\title{
READERS' COMMENTS
}

For personal use only. Not to be reproduced without permission of the publisher (editorial@gabi-journal.net).

Pro-generics policies and the backlog in medicines registration in South Africa: implications for access to essential and affordable medicines

Generics and Biosimilars Initiative Journal (GaBI Journal). 2015;4(2):58-63. doi:10.5639/gabij.2015.0402.014

\section{Comment by Ernest Schay, Bayer}

Thank you for this very interesting article. From a veterinary medicine perspective, it would certainly explain why such medicines are also experiencing a delay, as veterinary medicines use the same P \& A (Pharmaceutical and Analytical Committee) resources as human medicines. Unfortunately, as veterinary medicines do not appear on the Essential Medicines List (EML), we cannot take advantage of the fast track application system.

\section{Author Response}

Thank you for your comment. Yes, you are quite correct. We hope that the situation for all types of medicines will improve when SAHPRA (South African Health Products Regulatory Authority) is established, hopefully next year. The new regulator will be better resourced since it will be able to collect and retain its own fees, which is currently not the case. Perhaps the industry can engage the regulator to establish a veterinary medicines unit incorporating both clinical and P \& A divisions separate from that for human medicines. If the number of veterinary medicines dossiers submitted per annum is sufficiently high it may justify such a unit. Anyway, I am sure the situation will improve in future.

\section{Comment by Graeme James, Sanofi South Africa}

Thank you for the opportunity to comment on your paper regarding backlog. I wish to raise a couple of discussion points:

1. Your paper specifically deals with a group of tracer medicines in order to establish whether the backlog in medicine registration was harming access to affordable medicine. My thoughts are that the tracer group, mainly represented 'older' generics. If one wants to establish if access is delayed, one should test it on the premise that if a patent had to expire tomorrow, would there be generics available? If not, then it is a direct result of backlog. Thus, it would be worth doing this exercise again with generic molecules that have recently had patent expiry.

\section{Author Response}

This is an interesting comment. It would also depend on whether applications for 'new generics' have been received by the regulator sufficiently long in advance to review the dossiers and clarify all shortcomings so that by the time the patent expires the product would be registered. It must also be remembered that the period from the time the review starts until the product is registered depends not only on the regulator but also the applicant who must respond within three months after receiving recommendations from expert committees, e.g. P \& A. There are indications that many do not stick to this timeline. The reality is that with the current capacity at the Medicines Control Council (MCC) and the large number of annual submissions ( $>1,200$ per annum) it is unlikely that many generics of new off-patent medicines would be registered even if the backlog did not exist. Consideration should also be given to the large number of poor quality dossiers that fail the screening process.

2. The present backlog to approval (based on the 2014 figures) is 65 months. This excludes the screening period (which is currently one year). According to Industry Task Group (ITG) minutes there is a 4-year backlog before a dossier is even sent to an evaluator (1-year screening and currently only distributing January to March 2012 submissions). This demonstrates the buge impact we are facing if we want products to reach the market at patent expiry.

\section{Author Response}

One of the chief reasons for this state of affairs is the expedited review process that allows practically every drug on the EML to qualify for fast track status regardless of how many generics of a particular drug have already been registered. Most applications for expedited review are generics. Certain classes of drugs also automatically qualify to be reviewed by this pathway irrespective of whether the applicant applied for it - antiretroviral, anticancer, anti-tuberculosis and antimalarial drugs. Technical screening is actually done by reviewers, so it is part of the evaluation process. I am not convinced about the four-year waiting period for a submission to be sent to a reviewer. One has to do a meaningful analysis first before drawing such a conclusion.

3. With regards to duplicate registrations, it is pleasing to see that the MCC has released a guideline on 'Multiple submission of the same application with different Proprietary Names'. This should remove some of the duplicate reviews that we are experiencing as Industry.

\section{Author Response}

Agreed

4. I would not think restricting the number of applications/ submissions an applicant can make will pass constitutional muster. One cannot restrict trade due to a capacity issue. This would also be seen as anticompetitive.

\section{Author Response}

I think it would be reasonable to expect an applicant to justify why it wants to submit more than three copies of the same dossier under different trade names. It can be accepted that one would be for the private market, another for the public market and the last for export. Beyond that it becomes difficult to understand unless it is to trade in registration certificates (dossier farming). Although the regulator may not be in a position to stop an applicant from submitting several applications of the same dossier, it could arguably expect an applicant to pay higher application fees for such multiple submissions. From a public health perspective submitting multiple dossiers of the same product application is not easily justifiable.

5. Industry would like to see a fee-for-service arrangement, with regulated timelines. The idea would be that should an evaluation take longer than what is regulated, then the applicant would be entitled to a rebate on their application fee. In this way the productivity of the Health Authority improves.

\section{Author Response}

I am sure this would be acceptable if the regulator has the required manpower. Of course, should an applicant not respond in time to expert committee recommendations, then the regulator should also be in a position to levy penalties.

6. In order to address the backlog, both Industry and the MCC (Medicines Regulatory Affairs and the reviewers) should meet and discuss ways to solve the issues. It will be imperative that an open mind is kept when trying to find new solutions. It will not help if parties are not willing to let go of ideas/principles from the past.

\section{Author Response}

Agreed. It should be a collaborative project. My understanding is that such a process has already started.

Copyright (C) 2015 Pro Pharma Communications International 